\title{
Testing ERBB2 p.L755S kinase domain mutation as a druggable target in a patient with advanced colorectal cancer
}

\author{
Kyaw L. Aung, ${ }^{1}$ Tracy L. Stockley, ${ }^{1,2,3}$ Stefano Serra, ${ }^{1,2,3}$ Suzanne Kamel-Reid, ${ }^{1,2,3}$ \\ Philippe L. Bedard, ${ }^{1,4}$ and Lillian L. Siu ${ }^{1,4}$ \\ ${ }^{1}$ Drug Development Program, Division of Medical Oncology and Hematology; Princess Margaret Cancer \\ Centre, University Health Network, Toronto, Ontario M5S 1A8, Canada; ${ }^{2}$ Laboratory Medicine Program, \\ Department of Pathology, University Health Network, Toronto, Ontario M5G 2C4, Canada; ${ }^{3}$ Department of \\ Laboratory Medicine and Pathobiology, University of Toronto, Ontario M5S 1A8, Canada; ${ }^{4}$ Department of \\ Medicine, University of Toronto, Toronto, Ontario M5S 1A8, Canada
}

Corresponding author: Lillian. Siu@uhn.on.ca

(c) 2016 Aung et al. This article is distributed under the terms of the Creative Commons Attribution-NonCommercial License, which permits reuse and redistribution, except for commercial purposes, provided that the original author and source are credited.

Ontology terms: neoplasm of the gastrointestinal tract

Published by Cold Spring Harbor Laboratory Press

doi: $10.1101 / \mathrm{mcs} . a 001016$
Abstract Recent advances in molecular profiling technologies allow genetic driver events in individual tumors to be identified. The hypothesis behind this ongoing molecular profiling effort is that improvement in patients' clinical outcomes will be achieved by inhibiting these discovered genetic driver events with matched targeted drugs. This hypothesis is currently being tested in oncology clinics with variable early results. Herein, we present our experience with a case of advanced colorectal cancer (CRC) with an ERBB2 p.L755S kinase domain mutation, a BRAF p.N581S mutation, and an APC p.Q1429fs mutation, together with a brief review of the literature describing the biological and clinical significance of ERRB2 kinase domain mutations in CRC. The patient was treated with trastuzumab combined with infusional 5-fluorouracil and leucovorin based on the presence of ERBB2 p.L755S kinase mutation in the tumor and based on the available evidence at the time when standard treatment options had been exhausted. However, there was no therapeutic response illustrating the challenges we face in managing patients with potentially targetable mutations where results from functional in vitro and in vivo studies lag behind those of genomic sequencing studies. Also lagging behind are clinical utility data from oncology clinics, hampering rapid therapeutic advances. Our case also highlights the logistical barriers associated with getting the most optimal therapeutic agents to the right patient in this era of personalized therapeutics based on cancer genomics.

\section{INTRODUCTION}

In patients with advanced colorectal cancer (CRC), median survival of $\sim 21 \mathrm{mo}$ is achieved by using 5-fluorouracil (5-Fu)-based combination chemotherapies, FOLFIRI (5-Fu/leucovorin/irinotecan), and FOLFOX (5-Fu/leucovorin/oxaliplatin) in a sequential manner (de Gramont et al. 2000; Douillard et al. 2000; Saltz et al. 2000; Tournigand et al. 2004). Addition of biological agents to chemotherapy (bevacizumab/aflibercept in nonselected populations and cetuximab/panitumumab in patients with KRAS/NRAS wild-type tumors) has also been shown to improve clinical outcomes including survival (Hurwitz et al. 2004; Van Cutsem 
COLD SPRING HARBOR Molecular Case Studies
A case of colorectal cancer with an ERBB2 p.L755S mutation

et al. 2009, 2012; Douillard et al. 2010, 2013). In chemotherapy refractory setting, regorafenib, an oral multikinase inhibitor, improved survival when compared with best supportive care (Grothey et al. 2013). However, despite the advances made in management of advanced CRC, beyond the selection of patients with RAS wild-type tumors for epidermal growth factor receptor (EGFR)-targeted therapies, personalized treatment options are still very much limited.

With recent advances in next-generation DNA sequencing (NGS) technologies, it is now feasible to perform molecular profiling of tumors within an acceptable time frame at a reasonable cost. As such, there are several ongoing molecular profiling studies using NGS across multiple tumor types to identify actionable genetic driver events in individual tumors. The hypothesis behind these studies is that improved clinical outcomes will be achieved by targeting these actionable genetic aberrations with matched targeted therapies. However, there are barriers in development of this new treatment paradigm, especially when results from functional in vitro and in vivo studies lag behind those of genomic sequencing studies and utility of genomic data in the oncology clinic is still very experimental. Herein, we present a case of metastatic rectal cancer with an ERBB2 p.L755S kinase mutation treated with trastuzumab and 5-Fu/leucovorin illustrating the challenges we face in development of personalized medicine based on genomic profiles.

\section{RESULTS}

\section{Clinical History}

A 35-yr-old male, who was treated with a laparoscopic low anterior resection in June 2008 for a stage I (pT2NOMO) KRAS and NRAS wild-type, moderately differentiated, microsatellite stable rectal adenocarcinoma, developed liver metastases in July 2011 and underwent a right hepatectomy followed by 6 mo of pseudoadjuvant FOLFOX chemotherapy. In September 2012, his disease recurred with new liver and lung metastases and was treated with FOLFIRI and bevacizumab for $\sim 10$ mo with an initial disease response. On disease progression in July 2013, he was treated with panitumumab and progressed rapidly. He declined regorafenib because of concerns around toxicities. During the course of his illness, his primary rectal tumor and liver metastases were profiled within the Princess Margaret Cancer Genomic Program (CGP) and three somatic mutations, APC c.4285delC (p.Gln1429fs/ p.Q1429fs), BRAF c.1742A>G (p.Asn581Ser/p.N581S), and ERBB2 c.2264T>C (p.Leu755Ser/p.L755S) were detected in the tumors (detailed in Genomic Analyses and Methods sections and summarized in Table 1). As the ERBB2 p.L755S kinase domain mutation is likely to be an activating mutation, he was subsequently treated with three weekly intravenous doses of trastuzumab $(8 \mathrm{mg} / \mathrm{kg}$ loading dose at the first cycle followed by $6 \mathrm{mg} / \mathrm{kg}$ at subsequent cycles), combined with infusional 5-Fu and leucovorin in

\begin{tabular}{|c|c|c|c|c|c|c|c|}
\hline Gene & $\begin{array}{l}\text { Chr } \\
\text { No. }\end{array}$ & $\begin{array}{l}\text { HGVS DNA } \\
\text { ref }\end{array}$ & $\begin{array}{l}\text { HGVS } \\
\text { protein ref }\end{array}$ & $\begin{array}{c}\text { dbSNP/ } \\
\text { dbVar ID }\end{array}$ & $\begin{array}{l}\text { Variant } \\
\text { type }\end{array}$ & Genotype & $\begin{array}{l}\text { Predicted } \\
\text { effect }\end{array}$ \\
\hline$A P C$ & 5 & c. $4285 \mathrm{delC}$ & p.Gln1429fs & Not listed & Deletion & Heterozygous & Not known \\
\hline$B R A F$ & 7 & c. $1742 A>G$ & p.Asn581Ser & rs121913370 & Substitution & Heterozygous & Not known \\
\hline ERBB2 & 17 & c. $2264 \mathrm{~T}>\mathrm{C}$ & p.Leu755Ser & rs121913470 & Substitution & Heterozygous & $\begin{array}{l}\text { ERBB2 } \\
\quad \text { kinase } \\
\text { activation }\end{array}$ \\
\hline
\end{tabular}

HGVS, Human Genome Variation Society; dbSNP, Database for Short Genetic Variations. 
COLD SPRING HARBOR Molecular Case Studies
A case of colorectal cancer with an ERBB2 p.L755S mutation
November 2013, but his liver disease progressed after two cycles. He then received ziv-aflibercept plus a selective angiopoietin-2 monoclonal antibody within a clinical trial. The treatment ceased in March 2014 because of poor tolerance. He continued with best supportive care and expired in August 2014.

\section{Genomic Analyses}

Patient's written consent was obtained for molecular profiling. All genomic analyses detailed here were performed within the Research Ethics Board approved Princess Margaret Cancer Genomic Program's clinical research studies (REB reference numbers 11-0962 and 12-0361) and in a laboratory accredited by the College of American Pathologists and certified to meet Clinical Laboratory Improvement Amendments. Formalin-fixed, paraffin-embedded (FFPE) sections from the primary rectal tumor resected in 2008 (sample 1), 2011 liver resection (sample 2), and a liver biopsy performed in October 2013 (sample 3) were profiled. Tumor cell content of the samples 1, 2, and 3 was assessed by a qualified pathologist using a hematoxylin and eosin slide from each sample. Profiling of paired germline DNA extracted from peripheral blood was also performed to differentiate somatic and germline variants.

In June 2013, profiling of sample 2 (tumor cell content $80 \%$ ) using matrix-assisted laser desorption/ionization time-of-flight (MALDI-TOF) mass spectrometry (detailed in the Methods section) showed a heterozygous somatic ERBB2 p.L755S kinase domain mutation with an allele frequency of 57\%. In October 2013, next-generation sequencing of Sample 3 (tumor cell content 80\%) using the Illumina TruSeq Amplicon Cancer Panel (detailed in the Methods section) revealed the known ERBB2 p.L755S mutation and two additional heterozygous somatic mutations, APC p.Q1429fs and BRAF p.N518S, that were not detectable previously by MALDI-TOF mass spectrometry because the custom panel we used did not include them. To investigate whether these mutations were present in the primary rectal tumor, sample 1 (tumor cell content 70\%) was profiled using Illumina TruSeq revealing the presence of all three identical mutations in the primary tumor. Interestingly, increases in allele frequencies of all three mutations were observed over time, suggesting temporal clonal progression (Table 2). The biological effect of detected mutations was predicted using algorithms as previously described (Sukhai et al. 2016). Two detected mutations, BRAF p.N581S

Table 2. Summary of mutations identified in serial tumor samples (S1, S2, and S3) using MALDI-TOF mass spectrometry and Illumina TruSeq sequencing and their allele frequencies

\begin{tabular}{|c|c|c|c|c|c|c|c|c|c|c|c|}
\hline \multirow[b]{2}{*}{$\begin{array}{l}\text { Mutations } \\
\text { identified }\end{array}$} & \multicolumn{2}{|c|}{ Methods } & \multicolumn{3}{|c|}{$\begin{array}{c}\text { Allele } \\
\text { frequency } \\
(\%)\end{array}$} & \multicolumn{3}{|c|}{$\begin{array}{l}\text { Target coverage at } \\
\text { variant site }\end{array}$} & \multicolumn{3}{|c|}{$\begin{array}{l}\text { Mean target } \\
\text { coverage }\end{array}$} \\
\hline & $\begin{array}{l}\text { MALDI- } \\
\text { TOF }\end{array}$ & TruSeq & S1 & $\mathrm{S} 2$ & S3 & S1 & S2 & S3 & S1 & $\mathrm{S} 2$ & S3 \\
\hline $\begin{array}{c}\text { APC c.4285delC } \\
\text { (p.Gln1429fs) }\end{array}$ & $\begin{array}{l}\text { Not tested } \\
\text { by panel }\end{array}$ & Detected & 52 & NA & 85 & $7378 x$ & NA & $6420 x$ & $5115 x$ & NA & $4332 x$ \\
\hline $\begin{array}{c}\text { BRAF c. } 1742 A>G \\
\text { (p.Asn581Ser) }\end{array}$ & $\begin{array}{l}\text { Not tested } \\
\text { by panel }\end{array}$ & Detected & 30 & NA & 42 & $8771 x$ & NA & $9260 x$ & $5115 x$ & NA & $4332 x$ \\
\hline $\begin{array}{c}\text { ERBB2c.2264T >C } \\
\text { (p.Leu755Ser) }\end{array}$ & Detected & Detected & 33 & 57 & 68 & $8171 x$ & NA & $7056 x$ & $5115 x$ & NA & $4332 x$ \\
\hline
\end{tabular}

S1, S2, and S3 represent sample 1 (primary rectal tumor collected in 2008), sample 2 (tumor from 2011 liver resection), and sample 3 (tumor from 2013 liver biopsy), respectively. S1 and S3 were profiled using Illumina TruSeq sequencing and S2 was profiled using MALDI-TOF mass spectrometry.

MALDI-TOF, matrix-assisted laser desorption/ionization time of flight; NA, not applicable. 
COLD SPRING HARBOR Molecular Case Studies
A case of colorectal cancer with an ERBB2 p.L755S mutation

and ERBB2 p.L755S, were verified by Sanger sequencing. As the APC mutation is not actionable, no attempt was made to verify it using an orthogonal technology.

\section{DISCUSSION}

Unlike data reported in a previous study where all three patients with ERBB2 mutant CRCs had a KRAS mutation (Lee et al. 2006), our case had an ERBB2 kinase domain mutation p.L755S with a rare BRAF p.N581S and an APC p.Q1429fs mutation. BRAF p.N581S and APC p.Q1429fs mutations are found infrequently in CRCs (http://cancer.sanger.ac.uk/ cosmic) and their clinical significance in this disease was not known. ERBB2, on the other hand, is mutated in 3\%-5\% of CRC (http://cancer.sanger.ac.uk/cosmic) (Cancer Genome Atlas Network 2012). Mutations (small insertions and missense mutations) in the kinase domain of the ERBB2 were observed across multiple solid tumors including CRCs (Lee et al. 2006), and these mutations were shown to activate ERBB2 (Herter-Sprie et al. 2013). It was also known that $\mathrm{L755}$ is one of the critical amino acid residues that determine resistance to lapatinib, a reversible blocker of EGFR and ERBB2 tyrosine kinases (Kancha et al. 2011). As lapatinib inhibits only the inactive conformation of ERBB2 kinase, p.L755 mutations stabilize the kinase in active conformation, thereby conferring resistance to lapatinib (Kancha et al. 2011). In contrast, irreversible ERBB2 and EGFR inhibitors such as neratinib, and irreversible pan-ERBB inhibitor such as afatinib, bind to active conformation of the ERBB2 kinase thus likely to be more effective for treating tumors with p.L755 mutations (Kancha et al. 2011; De Greve et al. 2012). However, it was not known whether p.L755 mutations sensitize to trastuzumab response in CRC at the time of molecular profiling.

Considering the best available evidence at the time, it would have been most logical to treat our patient with an irreversible ERBB2 inhibitor. However, when this patient progressed on standard therapies, there was no matched clinical trial with an irreversible ERBB2 inhibitor. Request for regulatory approval via a Special Access Program for neratinib was declined because it was not licensed for use outside of clinical trial setting at the time in Canada. There was also no access to off-label use of approved agents such as afatinib. He was eventually treated with trastuzumab combined with infusional 5-fluorouracil and leucovorin chemotherapy (paid for by our research fund) based on evidence that in non-small-cell lung cancer with ERBB2 kinase domain mutations (mainly exon 20 insertions), partial responses were seen in nine of 15 patients (60\%) treated with trastuzumab plus chemotherapy (Mazieres et al. 2013).

Although there was evidence showing that combination treatment of pertuzumab and lapatinib produced sustained tumor regression in quadruple-negative (negative for activating mutations in KRAS, NRAS, BRAF, or PIK3CA), ERBB2-amplified, cetuximab-resistant CRC patient-derived xenografts (Bertotti et al. 2011), our case was considered to have an ERBB2activating kinase domain mutation rather than amplification, and as such combination treatment of pertuzumab or trastuzumab and lapatinib was not thought to be logical in our patient. Immunohistochemistry examination of the patient's resected primary cancer indeed showed that HER-2 immunohistochemical staining was zero, confirming that ERBB2 was not amplified in this case. The biological significance of the BRAF p.N581S mutation was also not known, and as such treatment with BRAF inhibitors or MEK inhibitors, either alone or in combination, was not considered.

The molecular profile of our patient is unique in that an ERBB2 p.L755S mutation was identified together with a BRAF p.N581S mutation. The biological and clinical significance of this unique profile was not known, and it was not possible to exploit this profile fully for genotype-matched therapy for our patient. Neratinib or another irreversible ERBB2 inhibitor was not accessible either because they were not approved for use outside of the clinical trial setting or not funded for off-license indications. It was not easily feasible to set up an $N$ of 1 
COLD SPRING HARBOR Molecular Case Studies
A case of colorectal cancer with an ERBB2 p.L755S mutation trial specifically for this individual case. Although trastuzumab was not the optimum drug for our patient, it was chosen mainly because of logistical reasons. This case clearly highlights the barriers of getting the optimum targeted drugs to patients in this era of personalized cancer therapeutics. These barriers, however, are not unique to Canada and require a wider global solution.

Since our patient was treated in 2013, there have been recent reports on the biological significance of ERBB2 kinase domain mutations in CRCs. These mutations, particularly p.L755S and p.V842I variants, are more frequently seen in Lynch or Lynch-like CRCs (Kloth et al. 2016) and are activating mutations in preclinical models (Kavuri et al. 2015; Kloth et al. 2016). There was no family history suggestive of Lynch syndrome in our patient and microsatellite status of our patient's tumor, tested by both fluorescent polymerase chain reaction (PCR) and immunohistochemistry, was stable. In vitro functional analyses indicate the superiority of more potent, irreversible pan-ERBB inhibitors over reversible ERBB inhibitors for inhibiting ERBB2 mutant CRC cell growth (Kloth et al. 2016). The ERBB2 p.L755S mutant CW2 cell line showed a strong sensitivity to irreversible pan-ERBB inhibitors but no sensitivity to lapatinib, cetuximab, or trastuzumab (Kloth et al. 2016). In CRC patient-derived xenografts, single-agent ERBB2-targeted therapy (trastuzumab, neratinib, or lapatinib) delayed the growth of ERBB2 p.S310Y and p.L866M mutant tumors, but durable tumor regression was only seen with dual ERBB2 blockade (trastuzumab combined with neratinib or lapatinib) (Kavuri et al. 2015). Failure of our patient's response to trastuzumab combined with 5-fluoroacil and leucovorin seems to concur with this emerging preclinical data. Activation of ERBB2 via mutations including P.L755S was also reported to mediate resistance to anti-EGFR therapy in vitro (Kavuri et al. 2015). The observation that our patient had primary resistance to panitumumab seems to anecdotally support this notion. However, no current preclinical evidence indicates that the BRAF p.N581S mutation contributed resistance to panitumumab or trastuzumab. Considering the preclinical results seen with CRC patient-derived xenografts, dual ERBB2 inhibition such as the combination of trastuzumab with neratinib may be more effective treatment for patients with ERBB2-mutated CRCs, but this hypothesis requires clinical evaluation.

\section{METHODS}

\section{DNA Extraction and Quantification}

DNA was extracted from FFPE tumor sections using the QIAamp DNA FFPE Tissue Kit (QIAGEN) and germline DNA from peripheral blood mononuclear cells (PBMCs) using automated extraction (MagAttract DNA Mini M48 kit; QIAGEN). DNA was quantified using the Qubit dsDNA Assay kit on the Qubit 2.0 Fluorometer (ThermoFisher Scientific).

\section{Mutation Profiling by MALDI-TOF Mass Spectrometry}

This analysis was performed using a custom panel to detect 279 mutations in 23 oncogenes as described elsewhere (Thomas et al. 2007). Briefly, DNA is amplified using a PCR primer mix, and a single base extension reaction is performed using extension primers that hybridize immediately adjacent to the mutation. From multiplexed reactions, peaks with different mass are resolved by MALDI-TOF mass spectrometry (MassARRAY, Agena Bioscience) using MassArray software as well as manual analysis. The lower limit of sensitivity for the assay is $10 \%-15 \%$. Neoplastic cells must be present at a minimum of $30 \%$ for mutation detection. 
CSH COLD SPRING HARBOR Molecular Case Studies
A case of colorectal cancer with an ERBB2 p.L755S mutation

\section{Illumina TruSeq Amplicon Cancer Panel Sequencing}

This was performed using the Illumina TruSeq Amplicon Cancer Panel (TSACP, Illumina), covering 212 amplicons in 48 cancer-associated genes (http://www.illumina.com/Docu ments/products/datasheets/datasheet_truseq_amplicon_cancer_panel.pdf), on the Illumina MiSeq sequencing platform. The postsequencing bioinformatics analysis pipeline using NeXTGene v2.3.1 software (SoftGenetics) allows the mapping of each amplicon to the reference human genome, the determination of coverage depth, and the identification of the sequence therein. Data were also visualized using the Integrative Genomics Viewer (IGV, Broad Institute). The lower limit of sensitivity for the assay is $5 \%-10 \%$.

\section{ADDITIONAL INFORMATION}

\section{Data Deposition and Access}

NGS data have been deposited at the European Genome-phenome Archive (EGA; https://www.ebi.ac.uk/ega/) under accession number EGAS00001001897. The reported variants have been submitted to COSMIC (Catalogue of Somatic Mutations in Cancer; http://cancer.sanger.ac.uk/cosmic). The variant ID numbers for ERBB2 p.L755S, BRAF p.N581S, and APC p.Q1429fs are COSM14060, COSM462, and COSM41625, respectively.

\section{Ethics Statement}

Molecular analyses were performed within the Research Ethics Board-approved Princess Margaret Cancer Genomic Program's clinical research studies (REB reference numbers 11-0962 and 12-0361) with the patient's written informed consent.

\section{Acknowledgments}

We thank our patient, his family, and the research staff of the Princess Margaret Cancer Genomics Program. We also thank Dr. Pratibha lyengar, Department of Pathology, The Credit Valley Hospital, Mississauga, Ontario, Canada, for performing HER-2 immunohistochemistry on our patient's tumor sample.

\section{Author Contributions}

All authors are members of the Princess Margaret Cancer Genomics Program. T.L.S. and S.K.-R. are responsible for accuracy of genomic data. K.L.A. and L.L.S. are responsible for the accuracy of clinical data. All authors contributed to writing the manuscript and approved the final manuscript.

\section{Funding}

The Princess Margaret Cancer Genomics Program was funded from the Princess Margaret Cancer Foundation (P.L.B., S.K.-R., T.L.S., and L.L.S.), a Cancer Care Ontario Applied Clinical Research Unit Grant (P.L.B., S.K.-R., and L.L.S.), a University of Toronto Division of Medical Oncology Strategic Innovation grant (P.L.B.), and an Ontario Ministry of Health and Long-Term Care Academic Health Sciences Centre Alternate Funding Plan Innovation Award (P.L.B.). The cost of trastuzumab was funded by the Princess Margaret Cancer Centre's Drug Development Program (L.L.S.).
Received February 16, 2016; accepted in revised form June 13, 2016.
Competing Interest Statement

The authors have declared no competing interest.

Aung et al. 2016 Cold Spring Harb Mol Case Stud 2: a001016 


\section{REFERENCES}

Bertotti A, Migliardi G, Galimi F, Sassi F, Torti D, Isella C, Cora D, Di Nicolantonio F, Buscarino M, Petti C, et al. 2011. A molecularly annotated platform of patient-derived xenografts ("xenopatients") identifies HER2 as an effective therapeutic target in cetuximab-resistant colorectal cancer. Cancer Discov 1: 508-523.

Cancer Genome Atlas Network. 2012. Comprehensive molecular characterization of human colon and rectal cancer. Nature 487: 330-337.

de Gramont A, Figer A, Seymour M, Homerin M, Hmissi A, Cassidy J, Boni C, Cortes-Funes H, Cervantes A, Freyer $G$, et al. 2000. Leucovorin and fluorouracil with or without oxaliplatin as first-line treatment in advanced colorectal cancer. J Clin Oncol 18: 2938-2947.

De Greve J, Teugels E, Geers C, Decoster L, Galdermans D, De Mey J, Everaert H, Umelo I, In't Veld P, Schallier D. 2012. Clinical activity of afatinib (BIBW 2992) in patients with lung adenocarcinoma with mutations in the kinase domain of HER2/neu. Lung Cancer 76: 123-127.

Douillard JY, Cunningham D, Roth AD, Navarro M, James RD, Karasek P, Jandik P, Iveson T, Carmichael J, Alakl M, et al. 2000. Irinotecan combined with fluorouracil compared with fluorouracil alone as first-line treatment for metastatic colorectal cancer: a multicentre randomised trial. Lancet 355: 1041-1047.

Douillard JY, Siena S, Cassidy J, Tabernero J, Burkes R, Barugel M, Humblet Y, Bodoky G, Cunningham D, Jassem J, et al. 2010. Randomized, phase III trial of panitumumab with infusional fluorouracil, leucovorin, and oxaliplatin (FOLFOX4) versus FOLFOX4 alone as first-line treatment in patients with previously untreated metastatic colorectal cancer: the PRIME study. J Clin Oncol 28: 4697-4705.

Douillard JY, Oliner KS, Siena S, Tabernero J, Burkes R, Barugel M, Humblet Y, Bodoky G, Cunningham D, Jassem J, et al. 2013. Panitumumab-FOLFOX4 treatment and RAS mutations in colorectal cancer. $N$ Engl J Med 369: 1023-1034.

Grothey A, Van Cutsem E, Sobrero A, Siena S, Falcone A, Ychou M, Humblet Y, Bouche O, Mineur L, Barone C, et al. 2013. Regorafenib monotherapy for previously treated metastatic colorectal cancer (CORRECT): an international, multicentre, randomised, placebo-controlled, phase 3 trial. Lancet 381: 303-312.

Herter-Sprie GS, Greulich H, Wong KK. 2013. Activating mutations in ERBB2 and their impact on diagnostics and treatment. Front Oncol 3: 86.

Hurwitz H, Fehrenbacher L, Novotny W, Cartwright T, Hainsworth J, Heim W, Berlin J, Baron A, Griffing S, Holmgren E, et al. 2004. Bevacizumab plus irinotecan, fluorouracil, and leucovorin for metastatic colorectal cancer. N Engl J Med 350: 2335-2342.

Kancha RK, von Bubnoff N, Bartosch N, Peschel C, Engh RA, Duyster J. 2011. Differential sensitivity of ERBB2 kinase domain mutations towards lapatinib. PLoS One 6: e26760.

Kavuri SM, Jain N, Galimi F, Cottino F, Leto SM, Migliardi G, Searleman AC, Shen W, Monsey J, Trusolino L, et al. 2015. HER2 activating mutations are targets for colorectal cancer treatment. Cancer Discov 5: 832-841.

Kloth M, Ruesseler V, Engel C, Koenig K, Peifer M, Mariotti E, Kuenstlinger H, Florin A, Rommerscheidt-Fuss U, Koitzsch U, et al. 2016. Activating ERBB2/HER2 mutations indicate susceptibility to pan-HER inhibitors in Lynch and Lynch-like colorectal cancer. Gut 65: 1296-1305.

Lee JW, Soung YH, Seo SH, Kim SY, Park CH, Wang YP, Park K, Nam SW, Park WS, Kim SH, et al. 2006. Somatic mutations of ERBB2 kinase domain in gastric, colorectal, and breast carcinomas. Clin Cancer Res 12:57-61.

Mazieres J, Peters S, Lepage B, Cortot AB, Barlesi F, Beau-Faller M, Besse B, Blons H, Mansuet-Lupo A, Urban T, et al. 2013. Lung cancer that harbors an HER2 mutation: epidemiologic characteristics and therapeutic perspectives. J Clin Oncol 31: 1997-2003.

Saltz LB, Cox JV, Blanke C, Rosen LS, Fehrenbacher L, Moore MJ, Maroun JA, Ackland SP, Locker PK, Pirotta N, et al. 2000. Irinotecan plus fluorouracil and leucovorin for metastatic colorectal cancer. Irinotecan Study Group. N Engl J Med 343: 905-914.

Sukhai MA, Craddock KJ, Thomas M, Hansen AR, Zhang T, Siu L, Bedard P, Stockley TL, Kamel-Reid S. 2016. A classification system for clinical relevance of somatic variants identified in molecular profiling of cancer. Genet Med 18: 128-136.

Thomas RK, Baker AC, Debiasi RM, Winckler W, Laframboise T, Lin WM, Wang M, Feng W, Zander T, MacConaill L, et al. 2007. High-throughput oncogene mutation profiling in human cancer. Nat Genet 39: 347-351.

Tournigand C, Andre T, Achille E, Lledo G, Flesh M, Mery-Mignard D, Quinaux E, Couteau C, Buyse M, Ganem G, et al. 2004. FOLFIRI followed by FOLFOX6 or the reverse sequence in advanced colorectal cancer: a randomized GERCOR study. J Clin Oncol 22: 229-237.

Van Cutsem E, Kohne CH, Hitre E, Zaluski J, Chang Chien CR, Makhson A, D'Haens G, Pinter T, Lim R, Bodoky $\mathrm{G}$, et al. 2009. Cetuximab and chemotherapy as initial treatment for metastatic colorectal cancer. N Engl J Med 360: 1408-1417.

Van Cutsem E, Tabernero J, Lakomy R, Prenen H, Prausova J, Macarulla T, Ruff P, van Hazel GA, Moiseyenko V, Ferry D, et al. 2012. Addition of aflibercept to fluorouracil, leucovorin, and irinotecan improves survival in a phase III randomized trial in patients with metastatic colorectal cancer previously treated with an oxaliplatin-based regimen. J Clin Oncol 30: 3499-3506. 


\title{
COLD SPRING HARBOR Molecular Case Studies
}

\section{Testing ERBB2 p.L755S kinase domain mutation as a druggable target in a patient with advanced colorectal cancer}

\author{
Kyaw L. Aung, Tracy L. Stockley, Stefano Serra, et al.
}

Cold Spring Harb Mol Case Stud 2016, 2: a001016 originally published online July 11, 2016 Access the most recent version at doi: $10.1101 / \mathrm{mcs} . a 001016$

References This article cites 21 articles, 9 of which can be accessed free at: http://molecularcasestudies.cshlp.org/content/2/5/a001016.full.html\#ref-list-1

License This article is distributed under the terms of the Creative Commons Attribution-NonCommercial License, which permits reuse and redistribution, except for commercial purposes, provided that the original author and source are credited.

Email Alerting Receive free email alerts when new articles cite this article - sign up in the box at the Service top right corner of the article or click here. 